Supporting Information for:

\title{
Tailoring Electric Field Standing Waves in Reflection-Absorption Infra-Red Spectroscopy to Enhance Absorbance in Buried Molecular Layers
}

Josée Maurais $^{1}$ and Patrick Ayotte ${ }^{1}$

${ }^{1}$ Département de chimie, Faculté des Sciences, Université de Sherbrooke, Sherbrooke (Qc), Canada 
A $2327 \mathrm{~nm}$ thick stratified composite film was tailored in order to meet first order destructive interference for the s-polarized component of the reflected IR-beam at $7.698 \mu \mathrm{m}$, the wavelength corresponding to the $\mathrm{HCH}$ bending mode (i.e., $1300.6 \mathrm{~cm}^{-1}$ ). The Me layer was deposited at selected z-positions from $z=0$ (i.e., Me layer located at the sample/substrate interface) to $z=$ total film thickness (i.e., Me layer located at the sample/vacuum interface) in $\sim 120 \mathrm{~nm}$ increments. Modulations in the $\mathrm{HCH}$ bending absorption feature intensity, resulting from the variation in the EFSW's amplitude, are observed in the RAIR spectra reported in Figure S1A for a $4 \mathrm{~nm}$ Me film adsorbed at $\mathrm{z}=0 \mathrm{~nm}, 989 \mathrm{~nm}$ and $2327 \mathrm{~nm}$. In order to quantify the depth profile of the EFSW amplitude, the experimental and simulated normalized integrated absorbance of the $\mathrm{HCH}$ bending vibrational excitations were reported at each z-position in Figure S1B for a $2327 \mathrm{~nm}$ total absolute film thickness (squares and continuous line, respectively).

One notices in Figure S1B, that strong modulations in the intensity of $\mathrm{HCH}$ bending spectral features as a function of z-position of the Me layer is observed. The decomposition of the EFSW amplitude into its contributions from the s-polarized and p-polarized components of the IR beam is particularly instructive, as it reveals important details of the dependance of $\mathrm{HCH}$ bending absorbance features amplitude on the Me layer z-position within the stratified composite films. Indeed, at $2327 \mathrm{~nm}$ film thickness, where maximum absorbance of the s-waves coincide with $\mathrm{HCH}$ bending excitations, maximum constructive interference conditions are met for the s-polarized component of the IR beam within the film thereby enhancing its contribution to the EFSW amplitude over that of the p-polarized component. This can be seen, in Figure S1C, to yield conditions that result in a strong modulation in the EFSW amplitude throught the sample. 

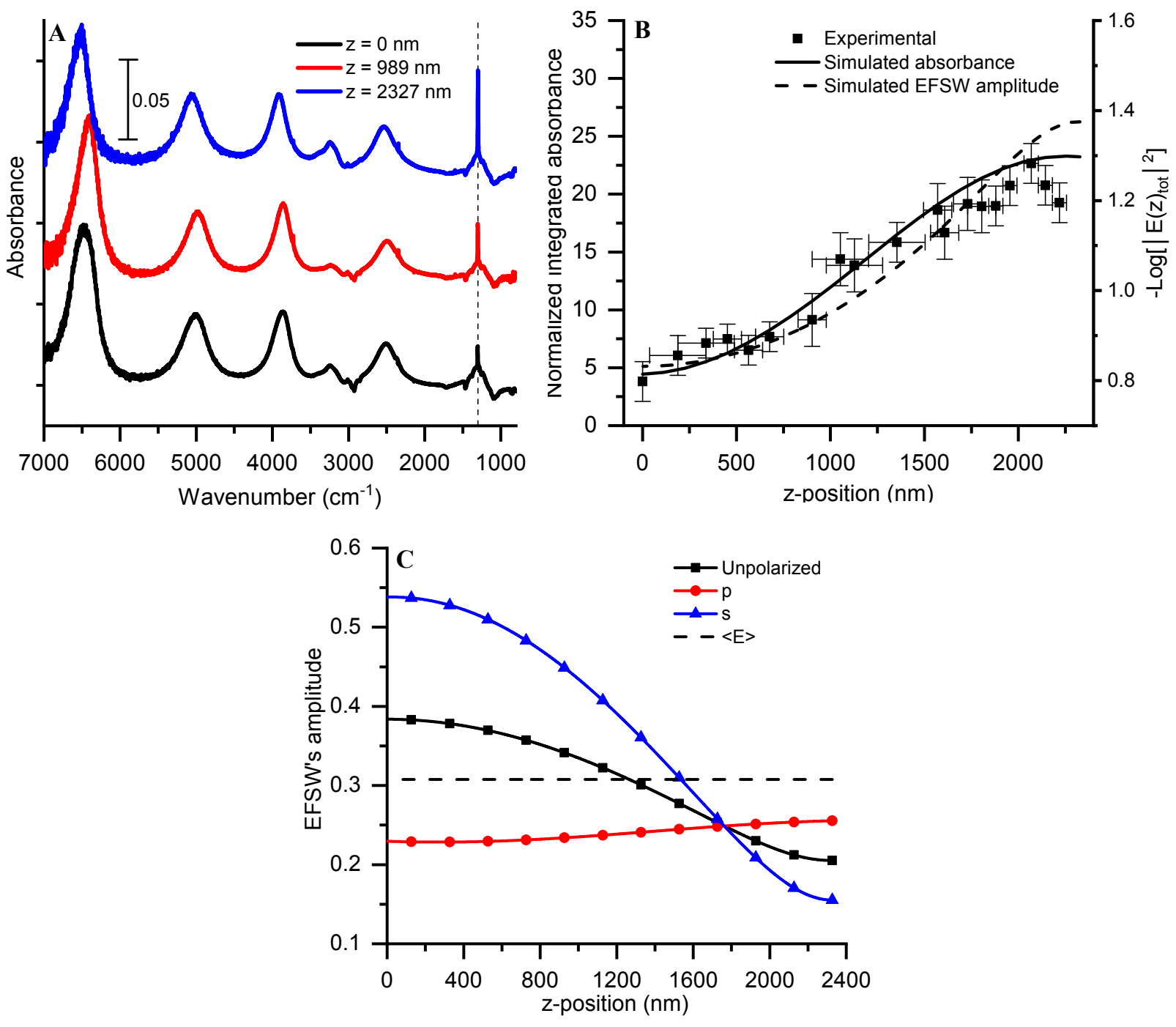

Figure S1. (A) Experimental spectra for stratified composite $\mathrm{Ar} / \mathrm{Me} / \mathrm{Ar}$ composite films composed of a $4 \mathrm{~nm}$ thick Me layer located at $\mathrm{z}=0 \mathrm{~nm}$ (black), $\mathrm{z}=989 \mathrm{~nm}$ (red) and $\mathrm{z}=2327 \mathrm{~nm}$ (blue) within a total sample thickness of $2327 \mathrm{~nm}$. (B) Normalized integrated absorbance for the $\mathrm{HCH}$ bending feature from the RAIR spectra of stratified $\mathrm{Ar} / \mathrm{Me} / \mathrm{Ar}$ composite films composed of a 4 $\mathrm{nm}$ thick Me layer as a function of its z-position within a total sample thickness of $2327 \mathrm{~nm}$ (black squares). Simulations of the RAIR spectra (continuous line), and of $-\log \left(\left|E(z)_{t o t}\right|^{2}\right)$, where $\left|E(z)_{t o t}\right|$ is the amplitude of the total electric field as a function of z-position (dashed lines), using the classical optics model, show how EFSW effects can yield strongly non-Beer-Lambert behavior in RAIR spectroscopy. (C) Contributions from the amplitude of the s-polarized (blue triangles) and p-polarized (red circles) components to the unpolarized EFSW amplitude (black squares) as a function of the depth profile (z) at a film thickness of $2327 \mathrm{~nm}$. The average amplitude of the unpolarized EFSW over the total film thickness is identified by the black dashed line. 